\title{
Perceptions of HIV Preexposure Prophylaxis Among Young Pregnant Women from Rural KwaZulu-Natal, South Africa
}

\author{
Laia Vazquez, MD, Anthony P. Moll, MBChB, ${ }^{2}$ Alexa Kacin, BS, ${ }^{3}$ \\ Ntombi Euginia Ndlovu, ${ }^{4}$ and Sheela V. Shenoi, MD, MPH ${ }^{5}$
}

\begin{abstract}
Young sub-Saharan women are at particularly high risk of HIV acquisition during pregnancy and the postpartum period and would potentially benefit from preexposure prophylaxis (PrEP). From June to August 2016, we interviewed 187 HIV negative pregnant women 18-24 years old in Tugela Ferry, Kwazulu-Natal province, a rural and among the poorest subdistricts in South Africa. Demographic data, HIV and PrEP knowledge, HIV risk, and readiness for oral tenofovir-based PrEP were collected using an information-motivation-behavior model-formatted instrument. Mean age was 20.3 years, 179 (95.7\%) were unemployed, and $137(73.3 \%)$ reported sex with one partner in the last month. Most were concerned that their sexual partner $(95.2 \%)$ potentially had HIV or had other sexual partners in the last month (36.4\%). Despite this, only 7 (3.7\%) women reported that condoms had been used consistently during sex; most $(97.3 \%)$ felt powerless to negotiate condom use with their partner. There was widespread interest in taking PrEP (97.3\%), and most women (>97\%) reported possessing the skills to take pills regularly, would commit to monthly visits, and were motivated to remain HIV negative to take care of their families. Young pregnant rural South African women are cognizant of their HIV risk and interested in prevention. Impending motherhood may portend increased interest in HIV prevention. We identified three potential obstacles to successful PrEP rollout among young pregnant women: hesitation about PrEP effectiveness (46\%), perceived HIV stigma (53.5\%), and risk compensation through decreased condom use $(9.6 \%)$. Comparative studies of motivations, skills, and rates of initiation and adherence among pregnant and nonpregnant women are needed to inform optimal implementation efforts.
\end{abstract}

Keywords: preexposure prophylaxis, HIV, pregnancy, primary prevention, South Africa, condom

\section{Introduction}

$\mathbf{O}$ RAL PREEXPOSURE PROPHYLAXIS, composed of $300 \mathrm{mg}$ of tenofovir disoproxil fumarate (TDF), alone or in combination with $200 \mathrm{mg}$ of emtricitabine (TDF-FTC), has been shown to be highly effective in preventing HIV infection among different populations when taken regularly. Based on the existing evidence, WHO released a technical brief in 2015 recommending preexposure prophylaxis (PrEP) to all persons at substantial risk of HIV infection (groups with an HIV incidence of about 3 per 100 person-years or higher). ${ }^{1}$ Unfortunately, young pregnant and lactating women from sub-Saharan Africa, who have one of the highest HIV infection rates globally, with an estimated HIV incidence of 3.8 per 100 woman-years, ${ }^{2}$ were excluded. This alarming rate, along with the associated risk of mother-to-child transmission, represents a public health emergency that needs immediate attention. ${ }^{2}$ Moreover, pregnancy and breastfeeding are associated with increased biological susceptibility to HIV-1 infection. ${ }^{3}$ Approval of PrEP for this population has been delayed due to safety concerns. The scarce data available on PrEP during pregnancy and breastfeeding suggest no significant differences in maternal and infant outcomes between women receiving PrEP or placebo at the time of conception, ${ }^{4-6}$ as well as minimal excretion of TDF into human breast milk. ${ }^{7}$ Data on pregnant and breastfeeding HIVpositive women show similar maternal, infant, and growth outcomes between antiretroviral therapy (ART) exposure (TDF and non-TDF containing regimens) and no ART; however, more information is needed regarding long-term

\footnotetext{
${ }^{1}$ Bridgeport Hospital Internal Medicine, Bridgeport, Connecticut.

${ }^{2}$ Church of Scotland Hospital, Tugela Ferry, South Africa.

${ }^{3}$ Northeastern University, Boston, Massachusetts.

${ }_{5}^{4}$ Philanjalo NGO, Tugela Ferry, South Africa.

${ }^{5}$ AIDS Program, Department of Medicine, Yale University School of Medicine, New Haven, Connecticut.
} 
safety. ${ }^{8-10}$ In light of this information, WHO now recommends initiation and continuation of PrEP in pregnant and lactating women at substantial risk of HIV infection, as part of a comprehensive program for prevention of mother-tochild transmission. ${ }^{8}$ Implementation science research is needed to understand the knowledge, perceptions, and beliefs about HIV infection and preventive strategies in this population. ${ }^{11}$ In this study we report on the perceptions about PrEP among young pregnant women from rural South Africa.

\section{Methods}

Tugela Ferry, KwaZulu-Natal province, South Africa, is characterized by extremely high HIV seroprevalence ( $>30 \%$ among women receiving prenatal care) rates. Tugela Ferry is the center of a rural impoverished subdistrict of 2000 square kilometers that is home to 180,000 traditional Zulu speaking people and is the third poorest subdistrict in South Africa. ${ }^{12}$ The topography is harsh: semiarid, rocky, and mountainous, with the population widely dispersed in isolated family compounds. The unemployment rate is $55 \%$, with $40 \%$ dependent on meager social welfare grants, with low levels of access to piped water $(1 \%)$ and electricity $(25 \%) ; 25 \%$ have no formal education. ${ }^{12}$ Truvada was approved for PrEP in South Africa in December 2015 and is available in selected locations for men who have sex with men (MSM) and sex workers. Other key populations, including adolescent girls and young women 1524 years old and individuals in serodiscordant relationships, are prioritized but do not yet have approval. South Africa has not yet approved PrEP for use in pregnant or lactating women.

From June to August 2016, local nurses at primary care clinics or the hospital maternity clinic identified young pregnant women 18-24 years old with documented negative HIV status, as eligible for the study. Trained research nurses confirmed eligibility and requested verbal consent to participate. An anonymous quantitative survey was administered by trained Zulu speaking research staff to young women in a private confidential location at the clinic. After the initial component of the survey, PrEP was described as a single antiretroviral pill, which if taken daily would successfully prevent them from acquiring HIV for as long as they took the medication, requiring initial clinical evaluation and blood work to establish eligibility. Information was collected on demographics, HIV knowledge and risk perceptions, and interest and readiness for oral TDF-based PrEP. Data were analyzed using Stata/IC, version 14.2.

\section{Results}

Among 187 young pregnant women interviewed, the mean age was 20.3 years (SD1.97), 93 (49.7\%) had completed secondary school, and the vast majority $(95.7 \%)$ was unemployed (Table 1). Most women (83.7\%) had a partner, and $73 \%$ reported one sexual partner in the last month. None of the women reported use of alcohol before sex or having ever been paid for sex.

While $185(98 \%)$ knew that HIV can be transmitted through sex and $184(95 \%)$ knew that condoms can reduce the risk, only $117(62.5 \%)$ knew that a woman can transmit HIV to her child and only $95(51 \%)$ knew that HIV can be transmitted through breast milk (Table 2). A total of 178 (95.2\%) participants reported that at least one of their sexual partners may have HIV, and $68(36.4 \%)$ believed that their
Table 1. Characteristics of Young Pregnant Women in Rural South Africa $(N=187)$

\begin{tabular}{lr}
\hline Age (mean $\pm S D)$ & $\begin{array}{c}20.27 \pm 1.9 \\
\mathrm{n}(\%)\end{array}$ \\
\hline $\begin{array}{l}\text { Site of interview } \\
\quad \text { Hospital }\end{array}$ & $84(44.9)$ \\
$\quad$ Primary Care Clinic & $103(55.1)$ \\
Marital status & \\
$\quad$ Married/partner & $160(84.1)$ \\
$\quad$ Single & $26(13.7)$ \\
Unemployed & $179(95.7)$ \\
Years of school attendance & $11(3.1)$ \\
$\quad 0-6$ & $88(47.6)$ \\
$7-11$ & $93(49.7)$ \\
$\quad \geq 12$ & $185(98.9)$ \\
Has had previous lifetime HIV testing & $137(73.3)$ \\
Has had sex with 1 partner in the past month
\end{tabular}

current sexual partner had been sexually active with other people in the last month. Despite this, 70 (37.4\%) women reported that condoms were never used by their male partners during sex, and $78(41.7 \%)$ reported that their partners used condoms less than half of the time. Only 7 (3.7\%) women reported that condoms were always used during sex. The vast majority $(182,97.3 \%)$ of women felt powerless to negotiate

Table 2. HIV Knowledge and Risk Among Young Pregnant South African Women $(N=187)$

Yes (\%)

Do you believe that HIV can be acquired $184(98.4)$ through unprotected sex with a person who has HIV?

Do you believe that HIV can be acquired through unprotected sex with a person who has HIV but who is on treatment?

Do you believe HIV can be transmitted through breast milk?

Do you believe an HIV positive mother can 117 (62.6) give her child HIV?

Do you believe condom use can reduce risk of 185 (98.9) HIV spread?

Do you worry about getting sick with HIV? 184 (96.8)

Do you believe any of your sexual partners has 178 (95.19) HIV?

If your sex partner does not want to use $182(97.3)$ condoms, there is little you can do about it

Frequency of condom use Never

$70(37.4)$

Less than $1 / 2$ time

$78(41.7)$ More than $1 / 2$ time

$36(19.2)$

It is easy to reject sexual advances from people who do not want to use condoms?

$62(33.2)$

In the heat of passion, you have a difficult time 62 (33.2) using a condom

Do you believe any of your sexual partners could have HIV?

Do you believe any of your sexual partners have had sex with other people in the past month?
$178(95.2)$

$68(36.4)$ 
condom use with their partner, and $62(33.3 \%)$ admitted having difficulty using condoms in the heat of the moment.

None in the study population knew about PrEP before the interview, but after a short introduction, $97.3 \%$ of women reported interest in taking oral PrEP (Table 3). Similarly, 97\% declared having the necessary skills to take pills, would start PrEP if a doctor recommended it, would take PrEP daily for as long as needed, and would commit to monthly visits to the primary care clinic to continue treatment. Overwhelmingly, respondents wanted to remain HIV negative to take care of their families $(99.5 \%)$ and were motivated to be a role model for others $(94.6 \%)$. The vast majority $(97.3 \%)$ believed that their families would support them if they decided to take PrEP. Some women (9.3\%) believed that by taking PrEP their partner may no longer need to use condoms. A small proportion (5.9\%) believed that by taking PrEP they may no longer need HIV screening, and 39 $(20.9 \%)$ were uncertain. We identified two other obstacles to PrEP implementation among young pregnant women: hesitation to take a medication that does not protect $100 \%$ from HIV infection $(86,46 \%)$, and concern about being mistaken

\section{Table 3. Readiness for Preexposure Prophylaxis Among Young Pregnant Rural South AFrICAN WOMEN $(N=187)$}

\begin{tabular}{lc}
\hline & Yes $(\%)$ \\
\hline Have you ever heard of PrEP? & $0(0)$ \\
Are you interested in taking PrEP? & $182(97.3)$
\end{tabular}

ArEP.

Their partner does not need to use a condom

You would no longer need to get screened for HIV

$18(9.6)$

$11(5.88)$

If you were taking PrEP...

Others would think less of you

Others would think you have HIV

You would feel comfortable telling others

Perceived benefits and barriers

Would you take PrEP even if it did not protect you $100 \%$ from getting HIV?

You prefer to avoid getting HIV by taking PrEP now

You would set a good example if you took meds to prevent HIV infection

Would you take PrEP, if you had to take it daily for it to work?

Would you take PrEP for as long as needed to 186 (99.5) prevent getting HIV?

Fear of side effects prevents you from being interested in taking PrEP

Life is so busy, you do not have time for medicine to prevent HIV

Queues in clinic are too long

Taking medications continuously is too expensive

$21(11.3)$

$100(53.5)$

$90(48.4)$

$86(46.0)$

$185(98.9)$

$177(94.6)$

$182(97.3)$

$6(3.2)$

$1(0.5)$

$117(62.6)$

$4(2.1)$

You would take PrEP if a doctor recommends 184 (98.4) it to you

You do not take any other medicines, so you do not want to start PrEP

My family would support me taking PrEP

You want to remain HIV negative to take care 1 of my family

PrEP, preexposure prophylaxis.
Table 4. Factors Associated with Young

Pregnant South African Women's Concerns about Being Mistaken as HIV Positive by Taking Preexposure Prophylaxis $(N=187)$

\begin{tabular}{|c|c|c|}
\hline & Yes $(\%)$ & $\mathrm{p}$ \\
\hline Being single & $26(13.7)$ & 0.02 \\
\hline $\mathrm{p}$ water & $120(64.2)$ & $<0.001$ \\
\hline Lack of electricity at home & $91(48.7)$ & 0.005 \\
\hline Attending school & $185(98.9)$ & 1.0 \\
\hline $\begin{array}{l}\text { Knowing someone who has been sick } \\
\text { with HIV }\end{array}$ & $134(71.7)$ & $<0.001$ \\
\hline $\begin{array}{l}\text { Difficulty using a condom in the heat of } \\
\text { the passion }\end{array}$ & $62(33.3)$ & $<0.001$ \\
\hline last month & $138(73.8)$ & 0.72 \\
\hline $\begin{array}{l}\text { Sexual partner has had sex with other } \\
\text { people in the past month }\end{array}$ & $68(36.4)$ & $<0.001$ \\
\hline Inconsistent con & 180 & 0.07 \\
\hline $\begin{array}{l}\text { Others will think less of you if you take } \\
\text { PrEP }\end{array}$ & 21 & 0.005 \\
\hline Others will avoid you if you take PrEP & & 0.001 \\
\hline $\begin{array}{l}\text { Not comfortable telling others about } \\
\text { PrEP }\end{array}$ & $95(50.8)$ & $<0.001$ \\
\hline $\begin{array}{l}\text { Not willing to share information with } \\
\text { others about PrEP }\end{array}$ & $14(7.5)$ & 0.04 \\
\hline port for taking PrEP & $182(97.3)$ & 0.89 \\
\hline nic are too long & $117(62.6)$ & $<0.001$ \\
\hline $\begin{array}{l}\text { Refusal to take PrEP if it does not } \\
\text { protect a } 100 \% \text { from HIV infection }\end{array}$ & $100(53.5)$ & $<0.001$ \\
\hline
\end{tabular}

PrEP, preexposure prophylaxis.

as HIV positive (53.5\%). Concerns about side effects $(3.2 \%)$ and costs $(5.9 \%)$ were present but less common.

Women concerned about being mistaken as HIV positive if taking PrEP (Table 4) were more likely to be single $(p=0.02)$, lack access to tap water $(p<0.001)$ and electricity $(p=0.005)$, believe that their partner had been sexually active with other people in the last month $(p<0.001)$, and believe that queues in the clinic are too long $(p<0.001)$. In addition, they were more likely to believe that others will avoid them $(p<0.001)$ or think less of them $(p<0.001)$. Women who knew someone who had been sick or died from HIV, relative or not, were more likely to be concerned about being mistaken as HIV positive $(p<0.001)$.

Women were more likely to disclose reasons for PrEP intake (Table 5) if they had been sexually active in the past month $(p=0.03)$ and if they believed that any of their sexual partners had HIV $(p=0.02)$. They were also more likely to

Table 5. Correlates of Disclosing Preexposure Prophylaxis Use Among Young Pregnant Women

\begin{tabular}{lrl}
\hline & Yes (\%) & \multicolumn{1}{c}{$\mathrm{p}$} \\
\hline Sex in the past month & $131(70)$ & 0.03 \\
$\begin{array}{l}\text { Concern that a sexual partner has HIV } \\
\text { People would not think less of me if I } \\
\quad \text { took PrEP }\end{array}$ & $21(11.2)$ & 0.004 \\
$\begin{array}{l}\text { People would not avoid me if I took } \\
\quad \text { PrEP }\end{array}$ & $5(2.7)$ & 0.04 \\
$\begin{array}{l}\text { People would not mistake me as HIV } \\
\text { positive if I took PrEP }\end{array}$ & $11(5.9)$ & 0.03 \\
& & \\
\end{tabular}

PrEP, preexposure prophylaxis. 
talk about their reasons to take PrEP if they believed that people would not think less of them $(p=0.004)$, would not avoid them $(p=0.04)$, or would not mistake them as HIV positive $(p=0.03)$.

\section{Discussion}

We present one of the first reports on understanding, interest, and concerns about PrEP among young pregnant women outside of known serodiscordant relationships in subSaharan Africa. Overall, the results of this study suggest that young pregnant women are cognizant of their HIV risk, express interest in protecting themselves and experience an inability to do so using traditional methods, and are potentially interested and willing to take oral TDF-based PrEP. A potentially major obstacle was perceived HIV stigma.

In our population of predominantly young, unmarried, and unemployed women, living in a rural impoverished region, widespread knowledge of sexual HIV transmission and preventive role of condoms did not translate into high levels of reported condom use. This happened despite concern of women about HIV infection and recognition of their risk of HIV acquisition from current sexual partners. Part of this paradox may be explained by the common practice of transactional sex $^{13}$ and age-discordant relationships ${ }^{14}$ in South Africa. Both phenomena amplify power imbalances and intimate partner violence already present in heterosexual relationships, limiting women's agency to negotiate safe sex and increasing their risk of HIV acquisition. ${ }^{15}$ Women may also desire childbearing, which can elevate women's social recognition. ${ }^{16}$

As seen in other studies carried out in the United States ${ }^{17}$ and Kenya, ${ }^{18,19}$ we identified great interest in PrEP among young HIV-negative pregnant women receiving antenatal care. Pregnant women in known serodiscordant relationships taking PrEP were enthusiastic although concerned with side effects that may impact their fetus. ${ }^{20}$ In our study, respondent characteristics that favor PrEP initiation include positive clinic experiences, appreciation for doctors' advice, high levels of motivation, family support, and self-reported ability to take daily oral TDF-based PrEP. Particularly encouraging are women's awareness of HIV risk and seeming ability to value future benefits of PrEP above immediate costs, which may differ from nonpregnant young women. ${ }^{21-23}$ Young pregnant women in our study were quite interested in taking PrEP and overwhelmingly motivated by remaining healthy and HIV negative to take care of their families, whereas a minority seemed concerned with PrEP side effects, adherence, and clinic visits. We speculate that impending motherhood could confer increased interest in future health outcomes, resulting in a potential lack of "present-bias.",23,24 This potential interest in long-term health may lend itself to greater interest in PrEP initiation and adherence. ${ }^{18,22,23}$ The differences between young women who are pregnant and those who are not with regard to motivation for PrEP need to be investigated in future studies.

We identified three main obstacles to PrEP acceptance: perceived stigma, hesitation about PrEP effectiveness, and potential for risk compensation. Perceived HIV stigma and external stigma are strong inhibitors to HIV care and may be particularly relevant barriers to PrEP implementation. ${ }^{25,26}$ Fear that PrEP would be misconstrued as HIV treatment and imply HIV positive status was a major concern among respondents and reinforces this finding in other studies as a potential barrier to PrEP implementation among pregnant women and women who are planning to have children. ${ }^{19,25,27}$ Fear of being perceived as HIV positive is concerning due to high levels of stigmatization in the community and personal relationships. Stigma was associated with having had contact with people affected by HIV, indicators of lower socioeconomic status, being single, and believing that their partner had been sexually active with others, suggesting that stigma may be experienced at greater levels by those in unstable relationships. Stigma was also accentuated in those who considered that clinic queues were too long, speculatively connoting decreased likelihood of engaging in existing health care services, as HIV stigma is prevalent among health care workers. $^{26,28}$

Further, we detected the potential for risk compensation, ${ }^{22,23}$ including cessation of condom use and HIV screening if taking PrEP, undermining the benefit of PrEP as part of a combination prevention package. Although multiple randomized controlled trials and observational studies in different populations have failed to confirm risk compensation behaviors, increased numbers of partners, decreased condom use, and increased sexually transmitted infections (STIs) have been recently noted in other high risk populations with increasing concern. ${ }^{29-31}$ In addition, those who were willing to discuss PrEP use were sexually active women who acknowledge that at least one of their partners could have HIV. Fear of being stigmatized could prevent women from sharing information about PrEP intake. PrEP implementation among pregnant women will need to consider incorporating strategies to address stigma, support adherence to optimize effectiveness, and prevent risk compensation.

The study also revealed deficient knowledge of vertical and breastfeeding HIV transmission risk, suggesting the need to revise prenatal health education as part of a comprehensive package of interventions to prevent mother-tochild transmission.

More information is needed to understand the needs and beliefs of young at-risk pregnant women in sub-Saharan Africa to ensure optimal HIV prevention, including potential use of and adherence to PrEP. Young nonpregnant women's perspectives on PrEP use are well delineated with respect to the mutuality framework of distrust, to uncertainty, alignment, and mutuality. ${ }^{32}$ Our results suggest that young pregnant women may have different attitudes toward PrEP, speculatively may feel greater trust in the product, less uncertainty about the benefit of PrEP in their lives, and more alignment and persistence compared to nonpregnant young women. ${ }^{32-34}$ However, while it is possible that pregnant women may experience a high degree of ownership over the use of PrEP, the substantive stigma noted in our study may detract from their overt support for PrEP. Comparative studies of pregnant and nonpregnant women are needed to determine how the potential fit with the mutuality framework informs potential strategies for optimal implementation in both populations. Risk assessment tools may be helpful to predict maternal HIV acquisition and, thus, identify pregnant and postpartum women who would benefit the most from PrEP. ${ }^{35}$ In addition, HIV stigma associated with oral PrEP formulations may be mitigated by alternative forms of PrEP delivery, such as vaginal rings ${ }^{36}$ and injectables. ${ }^{37}$ 
Our findings must be interpreted considering certain limitations. This is a quantitative dataset obtained from a crosssectional study and did not incorporate qualitative methods which likely could have provided additional insight. ${ }^{19,20,27}$ Next, we did not assess for physical or intimate partner violence or other potential consequences from their male partners as a result of PrEP use. In addition, these results may not be generalizable to other high-risk groups, including sex workers or nonpregnant adolescent girls and young women, but focus solely on young pregnant women not known to be in serodiscordant relationships. Further, as this study was done before approval of Truvada for prevention in South Africa, most women had not previously heard of PrEP and so the information gleaned during these interviews was first impressions. As South Africa proceeds with implementation and public education about PrEP increases, stigma may decrease. In addition, self-reporting does not necessarily predict actual initiation and adherence to $\operatorname{PrEP},{ }^{4}$ and therefore, we cannot predict whether these women who expressed interest would actually start and take PrEP daily if offered. Finally, we acknowledge that some women could have provided us with the most socially desirable response ${ }^{16}$ instead of their true opinions.

In summary, young pregnant women in a rural impoverished region of South Africa are cognizant of their HIV risk, report lack of agency around condom use, and are interested in protecting themselves using oral TDF-based PrEP. Respondents were overwhelmingly future oriented, possibly due to impending motherhood. HIV stigma, concern about PrEP effectiveness, and potential for risk compensation were identified as possible barriers to successful rollout in this group. Attention is needed to identify the optimal approaches toward PrEP implementation among pregnant and postpartum women in resource-limited settings.

\section{Acknowledgments}

Funding for the study came from NIAID (SS, \#K23AI089260), Gilead Foundation (SS, \#RO8290), and Doris Duke Fund to Retain Clinical Scientists/YCCI (SS, \#2015216). The authors acknowledge the diligent health care workers at Church of Scotland Hospital and Philanjalo NGO who strive to provide quality care for the Msinga community.

\section{Author Disclosure Statement}

The authors declare that they have no competing interests. SS's spouse performs part-time contract work for Amgen Pharmaceuticals. There is no conflict of interest regarding this article but it is included for full disclosure.

\section{References}

1. WHO Guidelines Approved by the Guidelines Review Committee. Guideline on When to Start Antiretroviral Therapy and on Pre-Exposure Prophylaxis for HIV. Geneva: World Health Organization Copyright ${ }^{\odot}, 2015$.

2. Mofenson LM. Tenofovir pre-exposure prophylaxis for pregnant and breastfeeding women at risk of HIV infection: The Time is Now. PLoS Med 2016;13:e1002133.

3. Thomson KA, Hughes J, Baeten JM, et al. Increased risk of HIV acquisition among women throughout pregnancy and during the postpartum period: A prospective per-coital-act analysis among women with HIV-1 infected partners. J Infect Dis 2018;218:16-25.

4. Marrazzo JM, Ramjee G, Richardson BA, et al. Tenofovirbased preexposure prophylaxis for HIV infection among African women. N Engl J Med 2015;372:509-518.

5. Van Damme L, Corneli A, Ahmed K, et al. Preexposure prophylaxis for HIV infection among African women. N Engl J Med 2012;367:411-422.

6. Mugo NR, Hong T, Celum C, et al. Pregnancy incidence and outcomes among women receiving preexposure prophylaxis for HIV prevention: A randomized clinical trial. JAMA 2014;312:362-371.

7. Mugwanya KK, Hendrix CW, Mugo NR, et al. Preexposure prophylaxis use by breastfeeding HIV-uninfected women: A prospective short-term study of antiretroviral excretion in breast milk and infant absorption. PLoS Med 2016;13:e1002132.

8. WHO Technical Brief: Preventing HIV During Pregnancy and Breastfeeding in the Context of PrEP. Licence: CC BYNC-SA 3.0 IGO. Geneva: World Health Organization Copyright $^{\odot}, 2017$.

9. Gibb DM, Kizito H, Russell EC, et al. Pregnancy and infant outcomes among HIV-infected women taking long-term ART with and without tenofovir in the DART trial. PLoS Med 2012;9:e1001217.

10. Nachega JB, Uthman OA, Mofenson LM, et al. Safety of tenofovir disoproxil fumarate-based antiretroviral therapy regimens in pregnancy for HIV-infected women and their infants: A systematic review and meta-analysis. J Acquir Immune Defic Syndr 2017;76:1-12.

11. Seidman DL, Weber S, Cohan D. Offering pre-exposure prophylaxis for HIV prevention to pregnant and postpartum women: A clinical approach. J Int AIDS Soc 2017; 20(Suppl 1):21295

12. Massyn NDC, Barron P, Haynes R, English R, Padarath A (ed). District Health Barometer 2011/12. Durban: Health Systems Trust, 2013.

13. Ranganathan M, MacPhail C, Pettifor A, et al. Young women's perceptions of transactional sex and sexual agency: A qualitative study in the context of rural South Africa. BMC Public Health 2017;17:666.

14. Maughan-Brown B, Evans M, George G. Sexual behaviour of men and women within age-disparate partnerships in South Africa: Implications for young women's HIV Risk. PLoS One 2016;11:e0159162.

15. Jewkes RK, Dunkle K, Nduna M, Shai N. Intimate partner violence, relationship power inequity, and incidence of HIV infection in young women in South Africa: A cohort study. Lancet 2010;376:41-48.

16. Todd CS, Stibich MA, Laher F, et al. Influence of culture on contraceptive utilization among HIV-positive women in Brazil, Kenya, and South Africa. AIDS Behav 2011;15: 454-468.

17. Seidman DL, Weber S, Timoney MT, et al. Use of HIV pre-exposure prophylaxis during the preconception, antepartum and postpartum periods at two United States medical centers. Am J Obstet Gynecol 2016;215:632.e1-632.e7.

18. Kinuthia J PJ, Mugwanya K, et al. High PrEP uptake among kenyan pregnant women offered PrEP during antenatal care. Conference on Retroviruses and Oportunistic Infections: Kenyatta National Hospital, 2018.

19. Pintye J, Beima-Sofie KM, Makabong'O PA, et al. HIVuninfected Kenyan adolescent and young women share 
perspectives on using pre-exposure prophylaxis during pregnancy. AIDS Patient Care STDS 2018;32:538-544.

20. Pintye J, Beima-Sofie KM, Kimemia G, et al. "I did not want to give birth to a child who has HIV": Experiences using PrEP during pregnancy among HIV-uninfected Kenyan Women in HIV-serodiscordant couples. J Acquir Immune Defic Syndr 2017;76:259-265.

21. Price JT, Rosenberg NE, Vansia D, et al. Predictors of HIV, HIV risk perception, and HIV worry among adolescent girls and young women in Lilongwe, Malawi. J Acquir Immune Defic Syndr 2018;77:53-63.

22. Celum CL, Delany-Moretlwe S, McConnell M, et al. Rethinking HIV prevention to prepare for oral PrEP implementation for young African women. J Int AIDS Soc 2015;18(4 Suppl 3):20227.

23. Linnemayr S, Stecher C. Behavioral economics matters for HIV research: The impact of behavioral biases on Adherence to Antiretrovirals (ARVs). AIDS Behav 2015;19: 2069-2075.

24. Linnemayr S. HIV prevention through the lens of behavioral economics. J Acquir Immune Defic Syndr 2015;68: e61-e63.

25. Calabrese SK, Dovidio JF, Tekeste M, et al. HIV preexposure prophylaxis stigma as a multidimensional barrier to uptake among women who attend planned parenthood. J Acquir Immune Defic Syndr 2018;79:46-53.

26. Calabrese SK, Earnshaw VA, Underhill K, et al. Prevention paradox: Medical students are less inclined to prescribe HIV pre-exposure prophylaxis for patients in highest need. J Int AIDS Soc 2018;21:e25147.

27. Bazzi AR, Leech AA, Biancarelli DL, Sullivan M, Drainoni ML. Experiences using pre-exposure prophylaxis for safer conception among HIV serodiscordant heterosexual couples in the United States. AIDS Patient Care STDS 2017;31:348-355.

28. Aggarwal S, Lee DH, Minteer WB, et al. Another generation of stigma? Assessing healthcare student perceptions of HIV-positive patients in Mwanza, Tanzania. AIDS Patient Care STDS 2017;31:87-95.

29. Fonner VA, Dalglish SL, Kennedy CE, et al. Effectiveness and safety of oral HIV preexposure prophylaxis for all populations. AIDS 2016;30:1973-1983.

30. Traeger MW, Schroeder S, Wright EJ, et al. Effects of preexposure prophylaxis for the prevention of human immu- nodeficiency virus infection on sexual risk behavior in men who have sex with men: A systematic review and metaanalysis. Clin Infect Dis 2018;67:676-686.

31. Newcomb ME MK, Feinstein BA, Forscher E, Mustanski B. Pre-exposure prophylaxis (PrEP) use and condomless anal sex: Evidence of risk compensation in a cohort of young men who have sex with men. JAIDS 2018;77:358364.

32. Amico KR, Wallace M, Bekker LG, et al. Experiences with HPTN 067/ADAPT study-provided open-label PrEP among women in Cape Town: Facilitators and barriers within a mutuality framework. AIDS Behav 2017;21:1361-1375.

33. Gill MM, Umutoni A, Hoffman HJ, et al. Understanding antiretroviral treatment adherence among HIV-positive women at four postpartum time intervals: Qualitative results from the Kabeho Study in Rwanda. AIDS Patient Care STDS 2017;31:153-166.

34. Nattey C, Jinga N, Mongwenyana C, et al. Understanding predictors of early antenatal care initiation in relationship to timing of HIV diagnosis in South Africa. AIDS Patient Care STDS 2018;32:251-256.

35. Pintye J, Drake AL, Kinuthia J, et al. A risk assessment tool for identifying pregnant and postpartum women who may benefit from preexposure prophylaxis. Clin Infect Dis 2017; 64:751-758.

36. Smith JM, Moss JA, Srinivasan P, et al. Novel multipurpose pod-intravaginal ring for the prevention of HIV, HSV, and unintended pregnancy: Pharmacokinetic evaluation in a macaque model. PLoS One 2017;12:e0185946.

37. Biello KB, Mimiaga MJ, Santostefano CM, Novak DS, Mayer KH. MSM at highest risk for HIV acquisition express greatest interest and preference for injectable antiretroviral PrEP compared to daily, oral medication. AIDS Behav 2018;22:1158-1164.

Address correspondence to:

Sheela V. Shenoi, MD, MPH

Yale University School of Medicine

135 College Street, Suite 323

New Haven, CT 06510

E-mail: sheela.shenoi@yale.edu 